\title{
A 20-week program of resistance or concurrent exercise improves symptoms of schizophrenia: results of a blind, randomized controlled trial
}

\author{
Bruna Andrade e Silva, ${ }^{1}$ Ricardo C. Cassilhas, ${ }^{2,3}$ Cecília Attux, ${ }^{4}$ Quirino Cordeiro, ${ }^{5}$ André L. Gadelha, ${ }^{4}$ \\ Bruno A. Telles, ${ }^{4}$ Rodrigo A. Bressan, ${ }^{4}$ Francine N. Ferreira, ${ }^{4}$ Paulo H. Rodstein, ${ }^{4}$ \\ Claudiane S. Daltio, ${ }^{4}$ Sérgio Tufik, ${ }^{6}$ Marco T. de Mello ${ }^{7}$ \\ ${ }^{1}$ Department of Nutrition, Universidade Federal de São Paulo (UNIFESP), São Paulo, SP, Brazil. ${ }^{2}$ Department of Physical Education, \\ Universidade Federal dos Vales do Jequitinhonha e Mucuri (UFVJM), Diamantina, MG, Brazil. ${ }^{3}$ Department of Psychobiology, UNIFESP, \\ São Paulo, SP, Brazil. ${ }^{4}$ Department of Psychiatry, UNIFESP, São Paulo, SP, Brazil. ${ }^{5}$ Faculdade de Ciências Médicas da Santa Casa de \\ São Paulo (FCMSCSP), São Paulo, SP, Brazil. ${ }^{6}$ Department of Psychobiology, UNIFESP, São Paulo, SP, Brazil. ${ }^{7}$ Universidade Federal de \\ Minas Gerais (UFMG), Belo Horizonte, MG, Brazil.
}

\begin{abstract}
Objective: To evaluate the effects of 20 weeks of resistance and concurrent training on psychotic and depressive symptoms, quality of life outcomes, and serum IGF-1, IGFBP-3, and brain-derived neurotrophic factor (BDNF) concentrations in patients with schizophrenia.

Methods: In this blind, randomized controlled clinical trial, 34 patients with schizophrenia were assigned to one of three groups: control (CTRL, $n=13$ ), resistance exercise (RESEX, $n=12$ ), or concurrent exercise (CONCEX, $n=9$ ). Symptoms, quality of life, strength, and other variables were assessed.

Results: A significant time-by-group interaction was found for the RESEX and CONCEX groups on the Positive and Negative Syndrome Scale (PANSS) total score for disease symptoms $(p=0.007)$, positive symptoms ( $p=0.003)$, and on the arm extension one-repetition maximum (1RM) test $(p=0.016)$. In addition, significant improvements on negative symptoms $(p=0.027)$, on the role-physical domain of the Short Form-36 Health Survey $(p=0.019)$, and on the chest press 1RM test $(p=0.040)$ were observed in the RESEX group. No changes were observed for the other variables investigated. Conclusions: In this sample of patients with schizophrenia, 20 weeks of resistance or concurrent exercise program improved disease symptoms, strength, and quality of life. ClinicalTrials.gov: NCT01674543.
\end{abstract}

Keywords: Physical exercise; IGF-1; BDNF; IGFBP-3; schizophrenia; resistance exercise; concurrent exercise

\section{Introduction}

Schizophrenia is a severe mental illness characterized by a combination of positive and negative symptoms. According to the DSM-IV, this disease is associated with social and work dysfunctions. ${ }^{1}$ Patients with schizophrenia also demonstrate a blunted affect and, typically, sedentary behavior, which can contribute to deterioration of symptoms and increase the risk of comorbidities. ${ }^{2,3} \mathrm{~A}$ recent systematic review demonstrated that patients with schizophrenia who participate less in physical activities have experience more negative symptoms, antipsychotic side effects, low selfefficacy, and other unhealthy lifestyle habits. ${ }^{4}$

The important mental and physical benefits of exercise in patients with schizophrenia include improvement of symptoms, ${ }^{5,6}$ a decreased need for care, ${ }^{7}$ improved quality of life, ${ }^{8}$ increased cardiorespiratory capacity, ${ }^{9}$ increased muscle strength, ${ }^{10}$ and increased functional capacity. ${ }^{5}$

Correspondence: Bruna Andrade e Silva, Rua Conselheiro Moreira de Barros, 1152/173C, CEP 02018-012, São Paulo, SP, Brazil. E-mail: brunaands @ hotmail.com

Submitted Oct 23 2014, accepted Feb 102015.
Studies of physical exercise among patients with schizophrenia have examined aerobic exercise, ${ }^{6,9,11}$ which is characterized by repeated rhythmic movements of the large muscle groups of the body, such as those in the arms and legs. Aerobic exercise mostly uses aerobic metabolism to produce energy; its adaptations are related to increases in maximum oxygen consumption $\left(\mathrm{VO}_{2 \max }\right)$ and the ability of skeletal muscles to generate energy via oxidative metabolism. ${ }^{12}$ However, little is known about the benefits of resistance training among patients with schizophrenia. Resistance training has been shown to increase muscle strength and muscle hypertrophy, as well as induce neuromuscular and functional adaptations. ${ }^{12,13}$ It has also been shown to benefit the mental health of other populations. Yerokhin et al. demonstrated that 10 weeks of resistance training increased the cognitive efficiency of patients with early dementia. ${ }^{14}$ Cassilhas et al. reported improvements in cognitive function, mood profile, anxiety, and quality of life among older adults after 6 months of resistance training. ${ }^{15,16}$

In addition to resistance training, previous studies of patients with schizophrenia have used a combination of aerobic and resistance exercises in the same session, which is known as concurrent exercise. ${ }^{5,17,18}$ Marzolini et al. examined concurrent exercise twice a week for 
12 weeks among patients with schizophrenia and found that this type of training improves general mental health and generally increases muscle strength. ${ }^{5}$ Scheewe et al. recently found similar results, with improvement of schizophrenia symptoms after 6 months of concurrent training. ${ }^{7}$ According to $\mathrm{Ho}$ et al., concurrent exercise had greater benefits for weight loss, fat loss, and cardiorespiratory fitness than aerobic or resistance exercise alone. ${ }^{19}$

The mechanisms through which physical exercise improves the symptoms of schizophrenia are not fully understood; however, a previous study showed that aerobic training increases hippocampal volume. ${ }^{6}$ This finding suggests that exercise improves the neuroplasticity and mental health of patients with schizophrenia. ${ }^{20}$ The molecular pathway activation of brain-derived neurotrophic factor (BDNF) and insulin growth factor-1 (IGF-1) are related to neuroplasticity. These markers can be measured both indirectly via serum, in humans, and directly from tissue, in animals. Numerous studies have indicated that activation of these pathways improves trophic communication between neurons, thereby improving neuroplasticity. ${ }^{21}$

Some studies have shown a connection between unbalance of growth factors and neurotrophins - especially BDNF, IGF-1, and IGF binding protein (IGFBP-3) and schizophrenia. ${ }^{22,23}$ In addition, schizophrenic patients exhibit reduced levels of BDNF and IGF-1. ${ }^{24,25}$ This finding was reinforced by Agid et al., who showed different pathways for BDNF/TrkB (tyrosine-related kinase B) and IGF-1/IGF-1R (insulin-like growth factor 1 receptor) in the neurotransmitter systems involved in schizophrenia, culminating in benefits in neuroplasticity. ${ }^{26}$ IGFBP-3, the most prevalent binding protein, carries most of the serum IGFs, regulating their actions and controlling their distribution to target tissues. The IGF-IGFBP-3 axis is involved in the pathogenesis of cardiovascular diseases. Patients with low circulating IGF-1 and high IGFBP-3 levels present increased risk of developing ischemic heart disease. ${ }^{27} \mathrm{~A}$ study that evaluated a program of dietary control and regular physical activity showed significant reductions in IGFBP-3 among obese patients taking clozapine for treatment of schizophrenia. ${ }^{28}$

Although resistance and concurrent exercise improve aspects of mental health, their effects remain unknown among adults with schizophrenia, for whom training variables (e.g., volume and intensity) are controlled, antipsychotic medications are monitored, and psychiatric scales are blinded. In addition, no studies have evaluated IGF-1, IGFBP-3, and BDNF after resistance or concurrent training among this population.

Therefore, the present study evaluated the effects of 20 weeks of resistance and concurrent training on psychotic and depressive symptoms and quality of life outcomes, as well as serum IGF-1, IGFBP-3, and BDNF concentrations, among patients with schizophrenia. We hypothesized that both exercise programs (resistance and concurrent) could cause improvement in the variables investigated.

\section{Methods}

This study was a blind, randomized controlled clinical trial. All study procedures were submitted to the Research
Ethics Committee of Universidade Federal de São Paulo (UNIFESP), São Paulo, Brazil, and approved under protocol number 249/10. Informed consent forms were obtained from participants, and this trial was registered at ClinicalTrials.gov with identifier NCT01674543.

\section{Sample}

Two clinics (Programa de Esquizofrenia [PROESQ], Centro de Atenção Psicossocial [CAPS], UNIFESP; and Faculdade de Ciências Médicas da Santa Casa de São Paulo) referred 47 adult males with a DSM-IV diagnosis of schizophrenia, aged 18 to 50 years (mean, 33.36 \pm 7.6 years), who were undergoing medical treatment. All participants underwent a physical examination and baseline electrocardiogram before testing. The study protocol was performed in a psychobiology and exercise research center. Physical education professionals, physiotherapists, nurses, and physicians were available at all times.

The patients were randomly assigned (www.randomizer.org) to one of three groups: control (CTRL), resistance exercise (RESEX), or concurrent exercise (CONCEX). The following eligibility criteria were adopted: a diagnosis of schizophrenia based on the DSM-IV criteria; clinically stable disease; stable doses of medication for at least 6 weeks; a sedentary lifestyle for more than 1 year; and clinical evaluation by a laboratory physician. Patients who did not attend at least $75 \%$ of the training sessions (i.e., 30 of 40 total training sessions) were excluded from the study. Certain patients were excluded (Figure 1) at the end of the study; the following sample of participants was considered in the analysis: CTRL $(n=13)$, RESEX $(n=12)$, and CONCEX $(n=9)$.

\section{Intervention}

Before training began, each participant completed three familiarization sessions during which they practiced the exercise to be performed during the training period. The interventions took place on Tuesdays and Thursdays for 20 weeks. Each session lasted 60 minutes and took place at the same time of day (between 1 p.m. and 5 p.m.), and each set was completed in approximately 30 seconds. Each training session was preceded by a 5-minute warmup on a Life Fitness ${ }^{\circledR}$ motorized treadmill at a constant velocity of $4 \mathrm{~km} / \mathrm{h}$. Professional physical educators monitored all exercise sessions.

CTRL sessions were held at the same place as the RESEX and CONCEX sessions and involved the same training protocol. However, the equipment load (i.e., the weight on each apparatus) was kept at the minimum, and the treadmill speed remained at $4 \mathrm{~km} / \mathrm{h}$ throughout the session. The patients executed two sets of 15 repetitions with a 1-minute rest for all equipment. We chose this control method to minimize confounders (e.g., social interaction and neuromuscular learning on the equipment) that might influence results.

Patients in the RESEX group were assigned a progressive resistance training program (Table 1), designed in accordance with the American College of Sports Medicine 


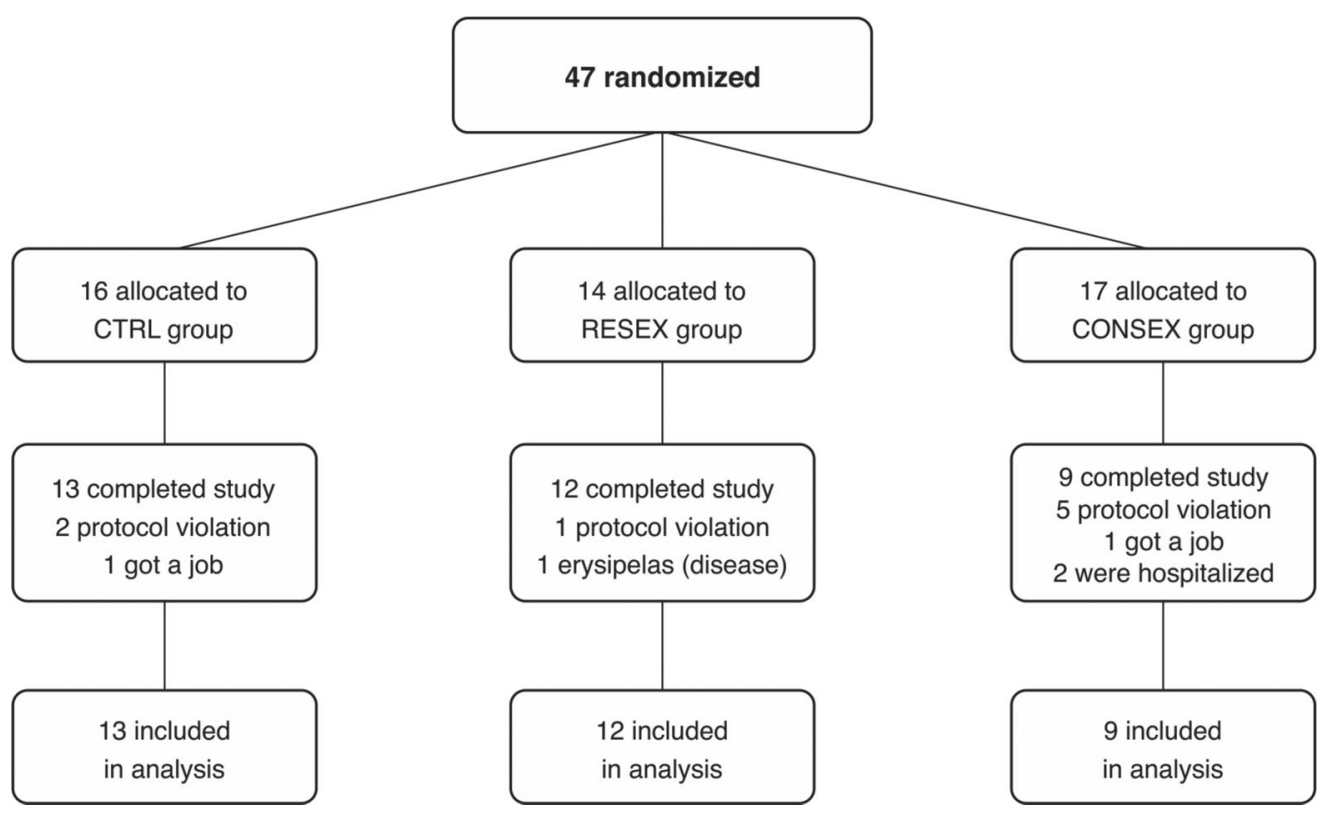

Figure 1 Flow diagram. CONSEX = concurrent exercise; CTRL = control; RESEX = resistance exercise.

guidelines concerning resistance training for adults. ${ }^{12}$ The exercises focused on the large muscle groups that are important for activities of daily living, and included the leg press, leg curl, vertical traction, chest press, arm extension, arm curl, and abdominal crunch. All exercises were performed on Technogym ${ }^{\circledR}$ equipment.

Patients in the CONCEX group were assigned endurance training and strength resistance exercises within the same session (Table 2). The resistance training matched that of the RESEX group; however, the training volume differed (Table 2). The aerobic program consisted of walking and running on a Life Fitness ${ }^{\circledR}$ treadmill with a prescription based on the variable $\mathrm{VO}_{2 \max }$. Heart rate was monitored throughout with a Polar ${ }^{\circledR}$ Vantage NV monitor.

\section{Measures}

Psychiatrists conducted individual, blind interviews of each patient at three points in time: baseline, week 10 , and week 20. The same psychiatrists interviewed the same patients, and all interviews were performed at the same time of day. The following scales and questionnaires were administered.

\section{Symptom severity}

The Positive and Negative Syndrome Scale (PANSS) was used to evaluate symptom severity via the general schizophrenia symptom score. ${ }^{29}$ The PANSS includes 30 items scored from 1 to 7 on a Likert scale; item scores are added to generate subscale and total scores. Higher scores indicate greater symptom severity. Scores range from 7 to 49 on the positive and negative subscales and from 16 to 112 on the general subscale.

\section{Medication monitoring}

Patients were instructed to notify the investigator of any change in their medications throughout the study. Antipsychotics were prescribed in cumulative doses and converted into chlorpromazine-mg/day equivalents as per Woods: clozapine, 50:100; olanzapine, 5:100; risperidone, 2:100; quetiapine, 75:100; and haloperidol, $2: 100 .^{30}$

Table 1 Protocol used for the resistance training group throughout the treatment period

\begin{tabular}{|c|c|c|c|}
\hline Stage & Intensity & Number of sets/repetitions & Interval \\
\hline Week 1 & $40 \% 1 \mathrm{RM}$ & 2 sets $/ 15$ repetitions & 2 minutes \\
\hline Week 2 & $50 \% 1 \mathrm{RM}$ & 2 sets $/ 15$ repetitions & 2 minutes \\
\hline Week 3 & $60 \% 1 \mathrm{RM}$ & 2 sets $/ 9-13$ repetitions & 1 minute \\
\hline Weeks 4,5 , and 6 & $70 \% 1 \mathrm{RM}$ & 2 sets $/ 8-12$ repetitions & 1 minute \\
\hline Weeks 7,8 , and 9 & $75 \% 1 \mathrm{RM}$ & 3 sets $/ 8-12$ repetitions & 1 minute \\
\hline Weeks 10,11 , and 12 & $75 \%$ 1RM & 3 sets $/ 6-8$ repetitions & 1 minute \\
\hline Weeks 13,14 & $80 \% 1 \mathrm{RM}$ & 3 sets $/ 6-8$ repetitions & 1 minute \\
\hline Weeks 15,16 & $80 \% 1 \mathrm{RM}$ & 3 sets $/ 6-8$ repetitions & 1 minute \\
\hline Weeks 17,18 & $85 \% 1 \mathrm{RM}$ & 3 sets $/ 6-8$ repetitions & 1 minute \\
\hline Weeks 19,20 & $85 \% 1 \mathrm{RM}$ & 3 sets $/ 6-8$ repetitions & 1 minute \\
\hline
\end{tabular}

$1 \mathrm{RM}=$ one-repetition maximum. 
Table 2 Protocol used for the concurrent training group throughout the treatment period

\begin{tabular}{|c|c|c|c|c|c|}
\hline \multirow[b]{2}{*}{ Stage } & \multicolumn{2}{|c|}{ Aerobic } & \multicolumn{3}{|c|}{ Resistance } \\
\hline & Intensity & Duration & Intensity & Number of sets/repetitions & Interval \\
\hline Week 1 & $40 \% \mathrm{VO}_{2 \max }$ & 25 minutes & $40 \% 1 \mathrm{RM}$ & 1 set/15 repetitions & 2 minutes \\
\hline Week 2 & $40 \% \mathrm{VO}_{2 \max }$ & 25 minutes & $50 \% 1 \mathrm{RM}$ & 1 set/15 repetitions & 2 minutes \\
\hline Week 3 & $50 \% \mathrm{VO}_{2 \max }$ & 25 minutes & $60 \% 1 \mathrm{RM}$ & 2 sets $/ 9-13$ repetitions & 1 minute \\
\hline Weeks $4,5,6$ & $50 \% \mathrm{VO}_{2 \max }$ & 25 minutes & $70 \% 1 \mathrm{RM}$ & 2 sets $/ 8-12$ repetitions & 1 minute \\
\hline Weeks $7,8,9$ & $60 \% \mathrm{VO}_{2 \max }$ & 25 minutes & $75 \% 1 \mathrm{RM}$ & 2 sets/8-12 repetitions & 1 minute \\
\hline Weeks $10,11,12$ & $60 \% \mathrm{VO}_{2 \max }$ & 25 minutes & $75 \% 1 \mathrm{RM}$ & 2 sets/6-8 repetitions & 1 minute \\
\hline Weeks 13, 14 & $70 \% \mathrm{VO}_{2 \max }$ & 25 minutes & $80 \% 1 \mathrm{RM}$ & 2 sets $/ 6-8$ repetitions & 1 minute \\
\hline Weeks 15,16 & $70 \% \mathrm{VO}_{2 \max }$ & 25 minutes & $80 \% 1 \mathrm{RM}$ & 2 sets $/ 6-8$ repetitions & 1 minute \\
\hline Weeks 17,18 & $75 \% \mathrm{VO}_{2 \max }$ & 25 minutes & $85 \% 1 \mathrm{RM}$ & 2 sets $/ 6-8$ repetitions & 1 minute \\
\hline Weeks 19, 20 & $75 \% \mathrm{VO}_{2 \max }$ & 25 minutes & $85 \% 1 \mathrm{RM}$ & 2 sets/6-8 repetitions & 1 minute \\
\hline
\end{tabular}

$1 \mathrm{RM}=$ one-repetition maximum; $\mathrm{VO}_{2 \max }=$ maximum oxygen consumption .

\section{Calgary Depression Scale for Schizophrenia (CDSS)}

Depressive symptoms were assessed using the Brazilian version of the CDSS. ${ }^{31}$ The CDSS is a semi-structured scale composed of nine items, all of which are defined using an operational criterion from 0 to 3 . This scale considers the preceding 2 weeks.

\section{Short Form-36 (SF-36) Health Survey}

The SF-36 quality of life questionnaire examines physical functioning and role limitations due to physical problems, vitality, bodily pain, and social functioning, as well as limitations due to emotional problems, mental health, and general health. SF-36 scores range from 0 to 100 , and higher scores indicate better health-related quality of life. The SF-36 exhibits acceptable reliability and validity among people with schizophrenia. ${ }^{32}$

\section{Biomarker measurements}

Three pre-prandial venous blood samples were obtained between 8:00 a.m. and 10:00 a.m. following a 10-h overnight fast for measurement of IGF-1, IGFBP-3, and BDNF. IGF-1 was quantitated in serum using enzymelinked immunosorbent assay (ELISA) via the E-20 DSL ${ }^{\circledR}$ Gênese ${ }^{\circledR}$ commercial kit. IGFBP-3 was measured using a chemiluminescent assay in an IMMULITE 2000 analyzer. BDNF serum concentrations were measured using Quantikine ${ }^{\circledR}$ ELISA kits for BDNF (R\&D Systems, Minneapolis, MN, USA), following the manufacturer-recommended procedure. The IGF-I/IGFBP-3 molar ratio was calculated based on $1 \mathrm{ng} / \mathrm{mL}$, IGF-I $=0.130 \mathrm{nmoL} I G F-I$ and $1-\mathrm{ng} / \mathrm{mL}$, IGFBP-3 $=0.036$ nmoL IGFBP-3. ${ }^{33}$

\section{Anthropometric measurements}

Body weight was measured with a Sanny ${ }^{\circledR}$ balance, and height with a stadiometer, both while patients were lightly clothed. Body mass index (BMI) was calculated as total body weight divided by height squared.

\section{Exercise testing}

Patients from all groups performed one-repetition maximum (1RM) tests for the resistance exercises (leg press, leg curl, vertical traction, chest press, arm extension, biceps curl, and abdominal crunch) on the same equipment used for training. These tests were performed at the same time of day as the training sessions. Prior to the $1 \mathrm{RM}$ test, all patients were assigned three training sessions 1 week before the intervention to become familiar with the equipment. The 1RM test protocol consisted of a 5-minute warm-up on a cycle ergometer followed by stretching exercises, then two series of 10 repetitions each on the test apparatus, the first with a light load and the second with a heavy load, followed by the first attempt at the test, increasing the load until the $1 \mathrm{RM}$ was reached. No more than three subsequent attempts were made, with 3-minute intervals between them. ${ }^{15}$

With regard to aerobic training, patients from the CONCEX group were assigned a progressive load test on a Life Fitness $9700 \mathrm{HR}$ treadmill. The protocol adopted for this test consisted of increasing speed by $1 \mathrm{~km} / \mathrm{h}$ per minute from $4 \mathrm{~km} / \mathrm{h}$ until a voluntary exhaustion maximum. Gas analysis (via ergospirometry) examined expired gases and directly measured $\mathrm{VO}_{2 \max }$. Tests were conducted in the laboratory under standard air conditioning and at the same time of day. As the sole objective of ergospirometry was to establish a parameter of aerobic intensity $\left(\mathrm{VO}_{2 \max }\right)$, it was only performed at baseline and in patients from the CONCEX group.

\section{Data analyses}

All statistical analyses were carried out in SPSS version 21.0. Multiple analyses of variance (ANOVAs) were used to examine between-group differences in demographics and clinical characteristics. Homogeneity of variance was assessed using Levene's test. The Greenhouse-Geisser correction was applied when the sphericity assumption (significant between-group differences in covariance) was violated; only the adjusted results were reported. A repeatedmeasures general linear model (GLM) with antipsychotic medications treated as a covariate was used to assess the intervention effects for all parameters, followed by Bonferroni's post-hoc test. P-values $<0.05$ were considered significant, and the eta square $\left(\eta^{2}\right)$ and power observed ( $p w$ ) were recorded. The data are presented in the tables as mean \pm standard error estimates. 
Table 3 Demographic and clinical characteristics of the sample

\begin{tabular}{lccc}
\hline & CTRL $(\mathrm{n}=13)$ & RESEX $(\mathrm{n}=12)$ & CONCEX $(\mathrm{n}=9)$ \\
\hline Age (years) & $33.36 \pm 12.19$ & $32.91 \pm 2.28$ & $33.55 \pm 2.63$ \\
Age of onset (years) & $20.61 \pm 1.73$ & $17.75 \pm 1.80$ & $22.44 \pm 2.08$ \\
Age initiation of treatment (years) & $20.62 \pm 5.65$ & $17.67 \pm 3.77$ & $22.56 \pm 7.84$ \\
Education (years) & $10.23 \pm 0.76$ & $11.25 \pm 0.80$ & $12.77 \pm 0.92$ \\
Weight $(\mathrm{kg})$ & $75.56 \pm 5.39$ & $83.27 \pm 5.61$ & $88.24 \pm 6.48$ \\
BMI $\left(\mathrm{kg} / \mathrm{m}^{2}\right)$ & $25.38 \pm 1.60$ & $27.98 \pm 1.67$ & $29.42 \pm 1.93$ \\
CGl score & $4.00 \pm 0.32$ & $3.75 \pm 0.33$ & $3.44 \pm 0.39$ \\
Antipsychotic medication dose, defined daily doses, CPZ & $1,107.92 \pm 114.05$ & $354.16 \pm 118.70^{*}$ & $538.88 \pm 137.07^{\dagger}$ \\
Antidepressant medication & 5 & 6 & 4 \\
Benzodiazepine & 3 & 2 & 1 \\
Mood stabilizer & 2 & 3 & \\
Anticholinergic & 3 & 2 & \\
\hline
\end{tabular}

Data presented as mean \pm standard error or $n$.

$\mathrm{BMI}=$ body mass index; CGI = Clinical Global Impression; CONCEX = concurrent training group; $\mathrm{CPZ}=$ chlorpromazine; CTRL = control group; RESEX = resistance training group.

General linear model one-way ANOVA with Bonferroni post-hoc comparison.

$*$ Differences between CTRL and RESEX group; ${ }^{\dagger}$ differences between RESEX and CONCEX group.

\section{Results}

Training session attendance exceeded $75 \%$ for all volunteers, and $27.65 \%$ of the sample dropped out. Demographic and clinical characteristics are shown in Table 3. All three groups were homogeneous at baseline, except with regard to antipsychotic medication dosage, which was significantly different among the groups $\left(F_{2,31}=\right.$ 11.323; $p=0.000 ; \eta^{2}=0.422 ; p w=0.987$ ); specifically, the CTRL group differed from the RESEX $(p<0.001)$ and CONCEX groups $(p=0.009)$.

Trends for the variables of interest from baseline through week 10 and week 20 are shown in Table 4. A significant time-by-group interaction was found with regard to PANSS total score (disease symptoms) after the intervention $\left(F_{2,31}=3.905 ; p=0.007 ; \eta^{2}=0.207\right.$; pw $=0.878$ ). In both the RESEX and CONCEX groups, disease symptoms improved after 10 weeks (RESEX; $p=0.002$; CONCEX; $p=0.026$ ) and 20 weeks (RESEX; $p \leqslant 0.001$; CONCEX: $p=0.003$ ), whereas in the CTRL group, they remained stable.

The trend observed for positive symptoms was similar to that observed for the total score, i.e., a significant timeby-group interaction $\left(F_{2,31}=4.455 ; p=0.003 ; \eta^{2}=0.229\right.$; pw $=0.920$ ). In the RESEX group, positive symptoms improved after 10 weeks $(p=0.039)$ and 20 weeks $(p \leqslant$ 0.001 ), whereas in the CONCEX group, these symptoms improved only after 20 weeks ( $p=0.016$ ); no change was found for the CTRL group. Differences were observed in negative symptoms with regard to the time-by-group interaction $\left(\mathrm{F}_{2,31}=2.965 ; \mathrm{p}=0.027 ; \eta^{2}=0.165 ; \mathrm{pw}=\right.$ 0.761 ); specifically, in the RESEX group, negative symptoms improved after 10 weeks $(p=0.001)$ and after 20 weeks $(p=0.002)$, compared to the baseline condition. No changes were observed in the other groups.

The time-by-group interaction also revealed significant SF-36 score changes with regard to physical role limitations $\left(F_{2,31}=3.200 ; p=0.019 ; \eta^{2}=0.176 ; p w=\right.$ 0.796). After 20 weeks of intervention, the RESEX group had improved significantly from baseline $(p=0.011)$, as had the CONCEX group $(p=0.014)$.
In general, strength for the 1RM test tended to increase in the RESEX group (Table 4); however, a significant time-by-group interaction was only observed for chest press $\left(F_{2,31}=2.679 ; p=0.040, \eta^{2}=0.152 ; p w=0.711\right)$. The RESEX group showed higher values than the CTRL group after 20 weeks $(p=0.043)$; increases in muscle strength were also found in the RESEX group over time, after 10 weeks $(p=0.003)$ and after 20 weeks $(p<$ 0.001), compared to the baseline condition. When comparing 10 and 20 weeks of training, significant differences were also found $(p=0.017)$. The arm extension 1RM test also revealed a significant time-bygroup interaction $\left(F_{2,31}=3.626, p=0.016, \eta^{2}=0.200\right.$; pw $=0.794)$, indicating increases in strength levels for the RESEX group after 10 weeks $(p \leqslant 0.001)$ and after 20 weeks $(p \leqslant 0.001)$, as well as in the CONCEX group, also after 10 weeks $(p=0.011)$ and after 20 weeks $(p=0.006)$.

\section{Discussion}

This study was the first blind, randomized controlled clinical trial to investigate the effects of a 20-week resistance and concurrent training protocol on schizophrenia symptoms, depression, and quality of life.

A 20-week resistance training and concurrent training program significantly improved the symptoms of patients with schizophrenia. After 10 and 20 weeks of exercise, patients in the RESEX group exhibited reductions in psychotic symptoms (positive, negative, and total scores) as measured by the PANSS, whereas a reduction in total and positive scores was observed for those from the CONCEX group only after 20 weeks. These encouraging results remained even after rigorous statistical treatments were applied to control for the effect of antipsychotic medications; this analysis increases the relevance of our results. These findings corroborate several previous studies that used yoga, relaxing exercises, walking, and running protocols. 6,34 Scheewe et al. assigned patients with schizophrenia to a concurrent training protocol composed primarily of cardiovascular exercises and three 


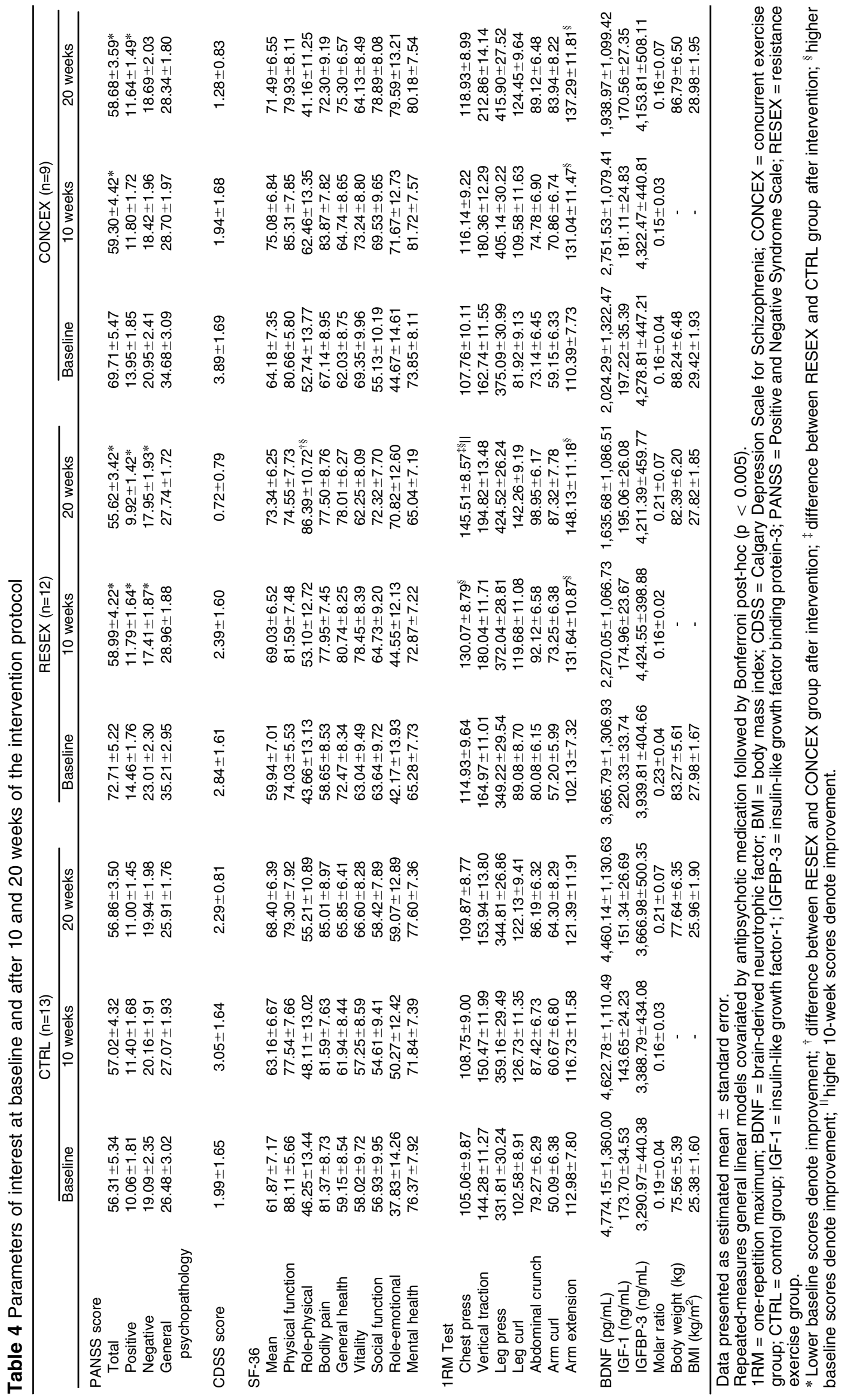


sets of 10 to 15 maximum repetitions of resistance exercises for six different muscle groups per week. ${ }^{35}$ These sessions lasted 1 hour and took place twice a week for 6 months. Their results showed a $20 \%$ decrease in PANSS total score and significant decreases in positive, negative, and other PANSS scores as compared with a control group undergoing occupational therapy.

No significant changes in CDSS or SF-36 scores were found in a previous study of high-intensity aerobic training administered three times per week for 8 weeks. ${ }^{9}$ This study was partially consistent with our results at week 10 . In addition, the groups might have had low levels of depression, thereby complicating any improvement.

An improvement in quality of life outcomes was observed in the RESEX group - specifically, in the role-physical domain of SF-36 - after intervention. Duraiswamy et al. assigned schizophrenic men to 20 weeks of yoga or walking intervention. ${ }^{34}$ At the end of the study, the authors observed improved quality of life outcomes for both groups. In other populations, improvement of quality of life outcomes have been observed in non-schizophrenic subjects who performed 6 months of resistance exercise. ${ }^{15}$

In addition to the psychiatric and quality of life benefits, higher muscle strength was demonstrated after both exercise programs (resistance and concurrent), as revealed by the $1 \mathrm{RM}$ test. Muscle strength maintenance is the basis for preserving functionality and independence in activities of daily living. ${ }^{10,36}$ A cohort study of 8,762 men aged 20 to 80 years showed that maximum force is independently and inversely associated with all-cause mortality. ${ }^{37} \mathrm{~A}$ resistance or concurrent training program might improve muscle strength and function.

The present study demonstrated that resistance and concurrent exercise have a therapeutic effect when provided as part of an interdisciplinary and continuous approach, as all volunteers used pharmacological treatments in addition to exercise-based treatment. Marzolini et al. also highlighted the importance of implementing resistance training as a therapeutic intervention in the treatment of schizophrenia. ${ }^{5}$ These authors also advocated the use of concurrent training to optimize the regulation of glucose metabolism, increase lean body mass, and improve body composition, as well as prevent the bone loss that is prevalent among patients with schizophrenia. $^{4,38}$

A recent pilot study assigned patients with schizophrenia to a maximum strength-training program for their legs to investigate whether this type of training would improve the mechanical efficiency of walking. The intervention protocol consisted of four sets of four repetitions of 85 to $90 \% 1 \mathrm{RM}$ leg-press exercises three times a week over 8 weeks. The results suggested that patients with schizophrenia can safely participate in and benefit from resistance training; furthermore, the program did improve the mechanical efficiency of walking. ${ }^{10}$

Serum BDNF and IGF-1 levels were measured as possible biomarkers of the neurobiological mechanisms of improvement or maintenance of schizophrenia symptoms thorough the exercise intervention. These trophic factors are the most commonly used biomarkers for this purpose, and play a critical role in signaling between various tissues, especially in the central nervous system. ${ }^{39,40}$ Moreover, they are involved in the etiopathogenesis of schizophrenia. ${ }^{25,41}$ These molecular pathway activators culminate in neuroplasticity, as well as decrease apoptosis and oxidative stress. ${ }^{21}$ However, no changes in these biomarkers were observed in any of the study groups. To the best of our knowledge, this was the first study to assess BDNF and IGF-1 levels after 20 weeks of resistance or concurrent exercise in schizophrenic patients. In line with these results, Krogh et al. recently found no changes in BDNF or IGF-1 in patients with major depression exposed to a 3-month aerobic exercise intervention. ${ }^{42}$ Other studies have also shown that resistance training does not increase serum concentrations of BDNF $^{43,44}$ but increases IGF $-1^{15}$ and that aerobic training increases serum BDNF. ${ }^{45,46}$ However, we found that BDNF and IGF-1 remained the same throughout the study period. No change in BDNF levels was expected for the RESEX groups, which is consistent with the results of Swift et al. ${ }^{43}$ An increase in BDNF levels was expected for the CONCEX group after aerobic training; however, this result did not occur.

Certain hypotheses can be considered with regard to BDNF. No significant anthropometric changes (i.e., weight loss with fat tissue reduction) were found in the present study. Other measures (e.g., nutritional counseling) are critical, as observed in a study of nutritional and exercise intervention over 6 months. ${ }^{28}$ Patient nutrition was not monitored in this study because it might have confounded our results, given that a decrease in IGFBP-3 and increases in the molar ratio of IGF-1 to IGFBP-3 can occur secondary to the improvement of metabolic status produced by the combination of diet and exercise. ${ }^{28}$ BDNF levels can also be elevated by reductions in body weight. ${ }^{47}$ This study did not observe changes in serum IGF-1 levels. This might be related to the drug treatments and energy balance, factors that can also influence neurotrophin functioning. ${ }^{48}$ Venkatasubramanian et al. found that antipsychotic-naive patients with schizophrenia had lower serum concentrations of IGF-1, which increased after the start of drug treatment. Two studies reported reductions in IGF-1 among patients taking clozapine $^{27,49}$; this phenomenon may have influenced the results of the present study.

In summary, our results suggest that resistance or concurrent exercise programs improve symptoms and the physical aspect of quality of life in patients with schizophrenia. This study reinforces the importance of resistance and concurrent training as non-pharmacological strategies for the treatment of schizophrenia.

The results of this study suggest that patients with schizophrenia should use resistance or concurrent training to improve their muscle strength and their physical, functional, and mental health. Two sessions per week with progressive loading are sufficient to produce benefits. Physical exercise is an important, low-cost therapeutic tool.

Study limitations include the small size of the sample, which comprised only males, and the fact that patients received different antipsychotic doses during the study, 
which may have masked the effects of exercise on symptoms and biomarker levels.

\section{Acknowledgements}

The authors are thankful to the Programa de Esquizofrenia (PROESQ), Centro de Atenção Psicossocial (CAPS), Faculdade de Ciências Médicas da Santa Casa de São Paulo, Fundação de Amparo è Pesquisa do Estado de São Paulo (FAPESP; postdoc grant 2013/ 05018-5), Associação Fundo de Incentivo è Pesquisa (AFIP), Centro de Estudos em Psicobiologia e Exercício (CEPE), Centro Multidisciplinar em Sonolência e Acidentes (CEMSA), Conselho Nacional de Desenvolvimento Científico e Tecnológico (CNPq), and Coordenação de Aperfeiçoamento de Pessoal de Nível Superior (CAPES).

\section{Disclosure}

The authors report no conflicts of interest.

\section{References}

1 American Psychiatric Association. Diagnostic and Statistical Manual of Mental Disorders, Fourth Edition, Text Revision (DSM-IV-TR). Arlington: American Psychiatric Publishing; 2000.

2 Faulkner G, Cohn T, Remington G. Validation of a physical activity assessment tool for individuals with schizophrenia. Schizophr Res. 2006;82:225-31.

3 Lindamer LA, McKibbin C, Norman GJ, Jordan L, Harrison K, Abeyesinhe $\mathrm{S}$, et al. Assessment of physical activity in middle-aged and older adults with schizophrenia. Schizophr Res. 2008;104:294-301.

4 Vancampfort D, Knapen J, Probst M, Scheewe T, Remans S, De Hert M. A systematic review of correlates of physical activity in patients with schizophrenia. Acta Psychiatr Scand. 2012;125:352-62.

5 Marzolini S, Jensen B, Melville P. Feasibility and effects of a groupbased resistance and aerobic exercise program for individuals with severe schizophrenia: A multidisciplinary approach. Ment Health Phys Act. 2009;2:29-36.

6 Pajonk FG, Wobrock T, Gruber O, Scherk H, Berner D, Kaizl I, et al. Hippocampal plasticity in response to exercise in schizophrenia. Arch Gen Psychiatry. 2010;67:133-43.

7 Scheewe TW, van Haren NE, Sarkisyan G, Schnack HG, Brouwer RM, de Glint M, et al. Exercise therapy, cardiorespiratory fitness and their effect on brain volumes: a randomised controlled trial in patients with schizophrenia and healthy controls. Eur Neuropsychopharmacol. 2013;23:675-85

8 Acil AA, Dogan S, Dogan O. The effects of physical exercises to mental state and quality of life in patients with schizophrenia. J Psychiatr Ment Health Nurs. 2008;15:808-15.

9 Heggelund J, Nilsberg GE, Hoff J, Morken G, Helgerud J. Effects of high aerobic intensity training in patients with schizophrenia: a controlled trial. Nord J Psychiatry. 2011;65:269-75.

10 Heggelund J, Morken G, Helgerud J, Nilsberg GE, Hoff J. Therapeutic effects of maximal strength training on walking efficiency in patients with schizophrenia - a pilot study. BMC Res Notes. 2012;5:344.

11 Beebe LH, Tian L, Morris N, Goodwin A, Allen SS, Kuldau J. Effects of exercise on mental and physical health parameters of persons with schizophrenia. Issues Ment Health Nurs. 2005;26:661-76.

12 Garber CE, Blissmer B, Deschenes MR, Franklin BA, Lamonte MJ, Lee IM, et al. American College of Sports Medicine position stand. Quantity and quality of exercise for developing and maintaining cardiorespiratory, musculoskeletal, and neuromotor fitness in apparently healthy adults: guidance for prescribing exercise. Med Sci Sports Exerc. 2011;43:1334-59.

13 Kamen G, Knight CA. Training-related adaptations in motor unit discharge rate in young and older adults. J Gerontol A Biol Sci Med Sci. 2004;59:1334-8.
14 Yerokhin V, Anderson-Hanley C, Hogan MJ, Dunnam M, Huber D, Osborne S, et al. Neuropsychological and neurophysiological effects of strengthening exercise for early dementia: a pilot study. Neuropsychol Dev Cogn B Aging Neuropsychol Cogn. 2012;19:380-401.

15 Cassilhas RC, Viana VA, Grassmann V, Santos RT, Santos RF, Tufik $S$, et al. The impact of resistance exercise on the cognitive function of the elderly. Med Sci Sports Exerc. 2007;39:1401-7.

16 Cassilhas RC, Antunes HK, Tufik S, de Mello MT. Mood, anxiety, and serum IGF-1 in elderly men given 24 weeks of high resistance exercise. Percept Mot Skills. 2010;110:265-76.

17 Poulin MJ, Chaput JP, Simard V, Vincent P, Bernier J, Gauthier Y, et al. Management of antipsychotic-induced weight gain: prospective naturalistic study of the effectiveness of a supervised exercise programme. Aust N Z J Psychiatry. 2007;41:980-9.

18 Vancampfort D, Probst M, Scheewe T, De Herdt A, Sweers K, Knapen J, et al. Relationships between physical fitness, physical activity, smoking and metabolic and mental health parameters in people with schizophrenia. Psychiatry Res. 2013;207:25-32.

19 Ho SS, Dhaliwal SS, Hills AP, Pal S. The effect of 12 weeks of aerobic, resistance or combination exercise training on cardiovascular risk factors in the overweight and obese in a randomized trial. BMC Public Health. 2012;12:704.

20 Scheewe TW, Takken T, Kahn RS, Cahn W, Backx FJ. Effects of exercise therapy on cardiorespiratory fitness in patients with schizophrenia. Med Sci Sports Exerc. 2012;44:1834-42.

21 Cassilhas RC, Lee KS, Fernandes J, Oliveira MG, Tufik S, Meeusen R, et al. Spatial memory is improved by aerobic and resistance exercise through divergent molecular mechanisms. Neuroscience. 2012;202: 309-17.

22 Autry AE, Monteggia LM. Brain-derived neurotrophic factor and neuropsychiatric disorders. Pharmacol Rev. 2012;64:238-58.

23 Akanji AO, Ohaeri JU, Al-Shammri SA, Fatania HR. Associations of blood levels of insulin-like growth factor (IGF)-I, IGF-II and IGF binding protein (IGFBP)-3 in schizophrenic Arab subjects. Clin Chem Lab Med. 2007;45:1229-31.

24 Martinotti G, Di lorio G, Marini S, Ricci V, De Berardis D, $\mathrm{Di}$ Giannantonio M. Nerve growth factor and brain-derived neurotrophic factor concentrations in schizophrenia: a review. J Biol Regul Homeost Agents. 2012;26:347-56.

25 Venkatasubramanian G, Chittiprol S, Neelakantachar N, Naveen MN, Thirthall J, Gangadhar BN, et al. Insulin and insulin-like growth factor-1 abnormalities in antipsychotic-naive schizophrenia. Am J Psychiatry. 2007;164:1557-60.

26 Agid Y, Buzsáki G, Diamond DM, Frackowiak R, Giedd J, Girault JA, et al. How can drug discovery for psychiatric disorders be improved? Nat Rev Drug Discov. 2007;6:189-201.

27 Wu MK, Huang CY, Liou YJ, Wang CK, Lee SD. Glucose-insulin homeostasis, lipid profiles and GH-IGF-IGFBP axis in clozapinetreated schizophrenic obesity versus non-psychiatric obesity. Int $\mathrm{J}$ Obes (Lond). 2008;32:436-42.

28 Wu MK, Wang CK, Bai YM, Huang CY, Lee SD. Outcomes of obese, clozapine-treated inpatients with schizophrenia placed on a sixmonth diet and physical activity program. Psychiatr Serv. 2007;58: 544-50.

29 Kay SR, Fiszbein A, Opler LA. The Positive and Negative Syndrome Scale (PANSS) for schizophrenia. Schizophr Bull. 1987;13:261-76.

30 Woods SW. Chlorpromazine equivalent doses for the newer atypical antipsychotics. J Clin Psychiatry. 2003;64:663-7.

31 Bressan RA, Chaves AC, Shirakawa I, de Mari J. Validity study of the Brazilian version of the Calgary Depression Scale for Schizophrenia. Schizophr Res. 1998;32:41-9.

32 Tunis SL, Croghan TW, Heilman DK, Johnstone BM, Obenchain RL. Reliability, validity, and application of the medical outcomes study 36item short-form health survey (SF-36) in schizophrenic patients treated with olanzapine versus haloperidol. Med Care. 1999;37:678-91.

33 Max JB, Limburg PJ, Ogunseitan A, Stolzenberg-Solomon RZ, Vierkant RA, Pollak MJ, et al. IGF-I, IGFBP-3, and IGF-I/IGFBP-3 ratio: no association with incident colorectal cancer in the AlphaTocopherol, Beta-Carotene Cancer Prevention Study. Cancer Epidemiol Biomarkers Prev. 2008;17:1832-4.

34 Duraiswamy G, Thirthalli J, Nagendra HR, Gangadhar BN. Yoga therapy as an add-on treatment in the management of patients with schizophrenia--a randomized controlled trial. Acta Psychiatr Scand. 2007; 116:226-32. 
35 Scheewe TW, Backx FJ, Takken T, Jörg F, van Strater AC, Kroes AG, et al. Exercise therapy improves mental and physical health in schizophrenia: a randomised controlled trial. Acta Psychiatr Scand. 2013;127:464-73.

36 Wildgust $\mathrm{HJ}$, Beary M. Are there modifiable risk factors which will reduce the excess mortality in schizophrenia? J Psychopharmacol. 2010;24:37-50.

37 Ruiz JR, Sui X, Lobelo F, Morrow JR Jr, Jackson AW, Sjöström M, et al. Association between muscular strength and mortality in men: prospective cohort study. BMJ. 2008;337:a439.

38 Meyer JM, Lehman D. Bone mineral density in male schizophrenia patients: a review. Ann Clin Psychiatry. 2006;18:43-8.

39 Cohen S, Greenberg ME. Communication between the synapse and the nucleus in neuronal development, plasticity, and disease. Annu Rev Cell Dev Biol. 2008;24:183-209.

40 Pan W, Kastin AJ. Interactions of IGF-1 with the blood-brain barrier in vivo and in situ. Neuroendocrinology. 2000;72:171-8.

41 Zhang XY, Liang J, Chen da C, Xiu MH, Yang FD, Kosten TA, et al. Low BDNF is associated with cognitive impairment in chronic patients with schizophrenia. Psychopharmacology (Berl). 2012;222:277-84.

42 Krogh J, Rostrup E, Thomsen C, Elfving B, Videbech P, Nordentoft M. The effect of exercise on hippocampal volume and neurotrophines in patients with major depression -- a randomized clinical trial. J Affect Disord. 2014;165:24-30.

43 Swift DL, Johannsen NM, Myers VH, Earnest CP, Smits JAJ, Blair SN, et al. The effect of exercise training modality on serum brain derived neurotrophic factor levels in individuals with type 2 diabetes. PLoS One. 2012;7:e42785.

44 Goekint M, De Pauw K, Roelands B, Njemini R, Bautmans I, Mets T, et al. Strength training does not influence serum brain-derived neurotrophic factor. Eur J Appl Physiol. 2010;110:285-93.

45 Babaei P, Azali Alamdari K, Soltani Tehrani B, Damirchi A. Effect of six weeks of endurance exercise and following detraining on serum brain derived neurotrophic factor and memory performance in middle aged males with metabolic syndrome. J Sports Med Phys Fitness. 2013:53:437-43.

46 Griffin ÉW, Mullally S, Foley C, Warmington SA, O'Mara SM, Kelly AM. Aerobic exercise improves hippocampal function and increases BDNF in the serum of young adult males. Physiol Behav. 2011;104:934-41.

47 Kuo F, Lee CH, Hsieh CH, Kuo P, Chen YC, Hung YJ. Lifestyle modification and behavior therapy effectively reduce body weight and increase serum level of brain-derived neurotrophic factor in obese non-diabetic patients with schizophrenia. Psychiatry Res. 2013; 209:150-4.

48 Venkatasubramanian G, Chittiprol S, Neelakantachar N, Shetty T, Gangadhar BN. Effect of antipsychotic treatment on Insulin-like Growth Factor-1 and cortisol in schizophrenia: a longitudinal study. Schizophr Res. 2010;119:131-7.

49 Melkersson KI, Hulting AL, Brismar KE. Different influences of classical antipsychotics and clozapine on glucose-insulin homeostasis in patients with schizophrenia or related psychoses. J Clin Psychiatry. 1999;60:783-91. 